\title{
The relationship between anogenital distance and the androgen receptor CAG repeat length
}

\author{
Michael L Eisenberg', Tung-Chin Hsieh², Alexander W Pastuszak², Matthew G McIntyre ${ }^{2}$, Rustin C Walters², \\ Dolores J Lamb ${ }^{2}$ and Larry I Lipshultz ${ }^{2}$
}

Anogenital distance (AGD) is used to define degree of virilization of genital development, with shorter length being associated with feminization and male infertility. The first exon of the androgen receptor (AR) consists of a polymorphic sequence of cytosine-adenineguanine (CAG) repeats, with longer CAG repeat lengths being associated with decreased receptor function. We sought to determine if there is an association between AGD and AR CAG repeat length. A cross-sectional, prospective cohort of men evaluated at a urology clinic at a single institution was recruited. AGD (the distance from the posterior scrotum to the anal verge) and penile length (PL) were measured. Sanger DNA sequence analysis was used to define CAG repeat length. AGD and CAG repeat lengths in 195 men were determined. On unadjusted analysis, there was no linear relationship between CAG repeat length and PL $(P=0.17)$ or AGD $(P=0.31)$. However, on sub-population analyses, those men with longer CAG repeat lengths $(>26)$ had significantly shorter AGDs compared to men with shorter CAG repeat lengths. For example, the mean AGD was 41.9 vs. $32.4 \mathrm{~mm}$ with a CAG repeat length $\leqslant 26$ vs. $>26$ $(P=0.01)$. In addition, when stratifying the cohort based on AGD, those with AGD less than the median (i.e. $40 \mathrm{~mm}$ ) had a longer CAG repeat length compared to men with an AGD $>40 \mathrm{~mm}(P=0.02)$. In summary, no linear relationship was found between AGD and AR CAG repeat length overall.

Asian Journal of Andrology (2013) 15, 286-289; doi:10.1038/aja.2012.126; published online 21 January 2013

Keywords: androgen receptor (AR); anogenital distance (AGD); genitalia; penile length (PL); perineum

\section{INTRODUCTION}

A marker for genital development, anogenital distance (AGD) has been examined in both animals and humans. ${ }^{1-4}$ In humans, a relationship among AGD, sperm count and testosterone production was described, suggesting a relationship between genital tract development and function. ${ }^{5-7}$ Nevertheless, the cause of varied AGDs between men remains uncertain. Rodent studies suggest that in utero androgen signaling during the masculinization programing window determines adult AGD with minimal influence from postnatal exposures to androgens. ${ }^{8}$ This correlation implies that AGD may reflect fetal determinants in the adult of prenatal androgen and estrogen exposures.

Although in utero environmental insults have been hypothesized to compromise normal human genital development, ${ }^{3,9,10}$ other intrinsic fetal factors may lead to abnormal testicular function and development. Androgen signaling is critical to the development of the male phenotype and the effects are mediated through the androgen receptor (AR). Thus, mutations in the AR may explain discrepancies in genital formation.

The AR is located on the short arm of the $\mathrm{X}$ chromosome and is encoded by eight exons. ${ }^{11}$ Exon 1 contains the polymorphic glutamine segment coded by the CAG repeat tract. The normal range for the CAG repeat tract of the AR is approximately 9-34 repeats and is known to vary with race and possibly fertility. ${ }^{11-13}$ The CAG repeat length is thought to correlate with androgen sensitivity, where shorter lengths display increased androgen sensitivity and longer lengths are more androgen-resistant, perhaps due to differential affinity of nuclear protein coactivators for the AR. ${ }^{14,15}$ Indeed, many groups have examined a relationship between the length of the polyglutamine repeat in the AR and male factor infertility ${ }^{16-18}$ To date, no work has examined the relationship between a man's AGD and his AR CAG repeat length.

\section{MATERIALS AND METHODS}

The methods of collection and cohort assembly were previously reported. ${ }^{5,7}$ Briefly, after obtaining Institutional Review Board approval from Baylor College of Medicine (USA), patients $(n=195)$ were recruited from a urology clinic specializing in reproductive and sexual medicine from August 2010 through October 2011. All men provided written consent for participation. Age, self-reported race, height and weight were recorded.

\section{Genital measurements}

The methods of genital measurement were previously described. ${ }^{5,7}$ Briefly, in the supine, frog-legged position with the legs abducted allowing the soles of the feet to meet, the distance from the posterior aspect of the scrotum to the anal verge was measured using a digital caliper (model No. 01407A; Neiko, USA). This was defined as the AGD.

From the same position, the stretched penile length (PL) was measured from the base of the dorsal surface of the penis to the tip of the glans.

\footnotetext{
${ }^{1}$ Department of Urology, Stanford University School of Medicine, Stanford, CA 94305, USA and 2Department of Urology, Baylor College of Medicine, Houston, TX 77030, USA Correspondence: Dr ML Eisenberg (eisenberg@stanford.edu)

Received: 17 August 2012; Revised: 10 September 2012; Accepted: 10 October 2012; Published online: 21 January 2013
} 
Table 1 Demographic, reproductive and anthropomorphic characteristics of the cohort $(N=195)$

\begin{tabular}{|c|c|c|}
\hline \multicolumn{2}{|l|}{ Characteristic } & \multirow{2}{*}{$\frac{\text { Value }}{45.1 \pm 14.4}$} \\
\hline Age (year), mean \pm s.d. & & \\
\hline \multirow[t]{2}{*}{ Race, $n(\%)$} & Caucasian & $151(77.4)$ \\
\hline & Others & $44(22.6)$ \\
\hline Height (cm), mean \pm s.d. & & $178.5 \pm 6.6$ \\
\hline Weight (kg), mean \pm s.d. & & $92.0 \pm 15.0$ \\
\hline \multirow[t]{3}{*}{ BMI category, $n(\%)$} & Normal & $28(14.4)$ \\
\hline & Overweight & $88(45.1)$ \\
\hline & Obese & $79(40.5)$ \\
\hline \multirow[t]{2}{*}{ Father, $n(\%)$} & No & $98(50.2)$ \\
\hline & Yes & $97(49.7)$ \\
\hline \multirow[t]{6}{*}{ Reason for office visit, $n(\%)^{a}$} & General urology & $21(10.9)$ \\
\hline & Erectile dysfunction & $10(5.2)$ \\
\hline & Hypogonadism & 64 (33.3) \\
\hline & Infertility & $75(39.1)$ \\
\hline & Vasectomy & $1(0.5)$ \\
\hline & Vasectomy reversal & $21(10.9)$ \\
\hline
\end{tabular}

Abbreviation: BMI, body mass index.

${ }^{a}$ Totals that add up to less than 195 indicate incomplete data.

AGD was measured by five investigators (four authors (MLE, TCH, MGM and RCW) and one collaborator). Previous data suggested reproducibility of the measurements with the correlation coefficient of 0.91 for both AGD and PL measurements. Moreover, the withinsubject standard deviation was $4.1 \mathrm{~mm}$ for AGD and $5.4 \mathrm{~mm}$ for stretched PL. In addition, there was no evidence for the measurement error being proportional to the magnitude of the measurement. ${ }^{5}$ Within-observer variability was not assessed in this measured population. However, investigators using a similar technique on paid volunteers reported relatively small variability $(2.1 \%-2.7 \%$ of the mean AGD). ${ }^{6}$ Moreover, previous measurements performed in children (MLE participated) also report good reproducibility (intraobserver coefficient of variation is 3.3). ${ }^{19}$

\section{DNA isolation and analysis}

After the clinical encounter, all participants had approximately $10 \mathrm{ml}$ of blood drawn. Genomic DNA was isolated from peripheral blood leukocytes using the Qiagen DNeasy Blood and Tissue Extraction kit (Qiagen, Inc., Valencia, CA, USA). The CAG repeat region of the AR was amplified by polymerase chain reaction using sequence-specific primers as previously described, and Sanger DNA sequencing performed by Genewiz, Inc. (South Plainfield, NJ, USA). ${ }^{17,20}$ The data were then analyzed using Mutation Surveyor (Softgenetics, Inc., State College, PA, USA) to calculate the number of CAG repeats in both the sense and antisense directions. Specimens were frozen at collection then all analyzed simultaneously at recruitment completion.

\section{Statistical analysis}

One-way analysis of variance (ANOVA) was used to compare normally distributed continuous variables. The Wilcoxon rank sum test was used to compare non-parametrically distributed continuous variables. Pearson's correlation coefficients were calculated to determine the association between continuous variables. Logistic regression models were used to determine the relationship between genital measures and ARCAG repeat length after dichotomizing CAG and AGD length based on experience gained from prior analyses conducted on AR CAG length. ${ }^{11}$ Covariates that have been shown to affect AGD were selected for inclusion a priori including age, race and fatherhood status. Models for PL were adjusted for age, race and body mass index (BMI). Given the non-parametric distribution of genital measures and CAG repeat length, regression models were also run with log-transformed variables with no differences in the overall conclusions. Effect modification of fatherhood was assessed using the likelihood ratio test by entering AGD along with fatherhood as well as the term for their product in the multivariable model. In addition, stratified analyses were also performed to judge effect modification. $P<0.05$ was considered significant, and all $P$ values were two sided. Analyses were performed using Stata 10 (StataCorp LP, College Station, TX, USA).

\section{RESULTS}

In all, 195 men had both AGD measured and AR CAG repeat length determined with a mean age (s.d.) of 45.1 (14.4) years. Men were seen for a variety of urological complaints. A majority of the men were Caucasian with equal numbers of fathers and childless men (Table 1). The mean number of CAG repeats was $21.7 \pm 3.3$. The mean AGD (s.d.) was 41.3 (13.4) $\mathrm{mm}$ and the mean PL (s.d.) was 113.2 (26.0) $\mathrm{mm}$.

Since the normal AR repeat length varies, we focused on each extreme. Men with longer CAG repeat lengths $(>26)$ had significantly

Table 2 AGDs stratified by CAG repeat length ${ }^{\mathrm{a}}$

\begin{tabular}{|c|c|c|c|c|c|c|c|}
\hline \multirow{2}{*}{$\begin{array}{l}\text { AR CAG repeat length } \\
\text { cutoff (repeat no.) }\end{array}$} & \multicolumn{2}{|r|}{$\leqslant$ cutoff } & \multicolumn{2}{|c|}{$>$ cutoff } & \multirow[b]{2}{*}{$P^{*}$} & \multicolumn{2}{|c|}{ Multivariable analysis ${ }^{b}$} \\
\hline & $\mathrm{n}$ & $\begin{array}{c}\text { Mean AGD (mm), } \\
\text { mean } \pm \text { s.d. }\end{array}$ & $\mathrm{n}$ & $\begin{array}{c}\text { Mean AGD (mm), } \\
\text { mean } \pm \text { s.d. }\end{array}$ & & OR $(95 \% \mathrm{Cl})$ & $P$ \\
\hline 15 & 6 & $45.1 \pm 12.6$ & 189 & $41.2 \pm 13.4$ & 0.41 & $1.15(0.64,2.05)$ & 0.64 \\
\hline 16 & 10 & $43.6 \pm 10.1$ & 185 & $41.2 \pm 13.6$ & 0.39 & $1.08(0.67,1.72)$ & 0.76 \\
\hline 17 & 18 & $41.3 \pm 9.4$ & 177 & $41.3 \pm 13.8$ & 0.70 & $0.87(0.58,1.31)$ & 0.51 \\
\hline 18 & 28 & $40.0 \pm 10.5$ & 167 & $41.5 \pm 13.8$ & 0.91 & $0.82(0.58,1.15)$ & 0.26 \\
\hline 19 & 42 & $42.5 \pm 13.1$ & 153 & $40.9 \pm 13.5$ & 0.34 & $1.04(0.79,1.36)$ & 0.80 \\
\hline 24 & 159 & $41.3 \pm 12.8$ & 36 & $41.4 \pm 15.8$ & 0.61 & $0.95(0.72,1.26)$ & 0.74 \\
\hline 25 & 176 & $41.8 \pm 13.4$ & 19 & $36.7 \pm 12.9$ & 0.09 & $1.41(0.91,2.17)$ & 0.12 \\
\hline 26 & 183 & $41.9 \pm 13.4$ & 12 & $32.4 \pm 10.9$ & 0.01 & $2.20(1.12,4.32)$ & 0.02 \\
\hline 27 & 188 & $41.7 \pm 13.4$ & 7 & $29.0 \pm 7.2$ & 0.01 & $4.00(1.22,13.11)$ & 0.02 \\
\hline 28 & 192 & $41.5 \pm 13.3$ & 3 & $28.0 \pm 10.4$ & 0.07 & $4.43(0.70,28.09)$ & 0.11 \\
\hline
\end{tabular}

Abbreviations: AGD, anogenital distance; CAG, cytosine-adenine-guanine; $\mathrm{Cl}$, confidence interval; OR, odds ratio.

${ }^{a}$ Listed lengths represent the extremes of long and short repeats.

${ }^{b}$ Multivariable OR $(95 \% \mathrm{Cl}$ ) adjusted for age, race and fatherhood states the odds for having a CAG repeat length less than the cutoff for each 10 mm increase in AGD.

* $P$ value represents Wilcoxon rank sum analyses. 


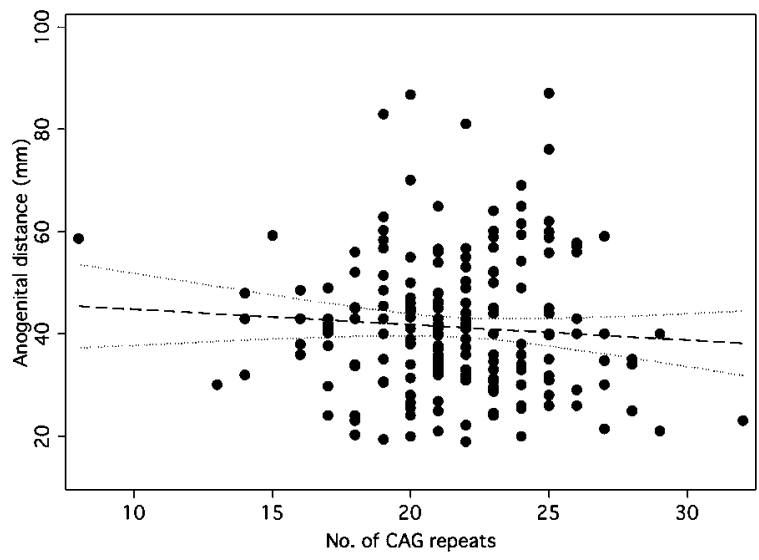

Figure 1 Scatterplot and linear best fit line (dashed line) with 95\% confidence interval (dotted line) demonstrating relationship between anogenital distance and CAG repeat length. $C A G$, cytosine-adenine-guanine.

shorter AGD compared to men with shorter CAG repeat lengths (Table 2). For example, the mean AGD was 41.9 vs. $32.4 \mathrm{~mm}$ with a CAG repeat length $\leqslant 26$ vs. $>26$. The relationship remained after multivariable adjustment. For short CAG repeat lengths, the AGD length differences were not significantly different (Table 2). There was no difference in AGD length across intermediate CAG repeat lengths. In addition, there was no linear relationship between AGD and the number of CAG repeats $(P=0.31$; Figure 1$)$.

When examining PL, there was no linear relationship between PL and CAG repeat length $(P=0.17)$, nor when examining longer or shorter CAG repeat lengths.

When stratifying the cohort based on AGD, those with AGD shorter than the median (i.e. $40 \mathrm{~mm}$ ) had a longer CAG repeat length compared to men with an AGD $>40 \mathrm{~mm}$ (22.3 vs. 21.1; $P=0.02$; Table 3; Figure 2). After adjusting for age, race and fatherhood status, the relationship remained whereby for each additional AR CAG repeat, the odds of a shorter AGD increased by $11 \%$ ( $95 \%$ confidence interval: 1-22\%; Table 3). A similar trend existed for other AGD lengths around the median AGD but none reached statistical significance (Table 3). In contrast, men with a PL longer and shorter than the median (116.5 mm) had similar CAG repeat lengths (21.8 vs. 21.5; $P=0.48$ ). After adjusting for age, race and BMI, no relationship was observed between PL and CAG repeat length (data not shown).

Of the 12 men with a CAG repeat length $>26$, only nine $(75 \%)$ had an AGD less than the median AGD for the group (40 mm). Of the seven men with a CAG repeat length $>27$, six $(86 \%)$ had an AGD less than the median. Of the three men with a CAG repeat length $>28$, all had an AGD less than the median AGD.
Of the 49 men with an AGD in the lowest quartile (AGD $<31 \mathrm{~mm}$ ), only six (12\%) had a CAG repeat length $>26$.

\section{DISCUSSION}

While no relationship was found between AGD and AR CAG repeat length overall, the current study suggests that men with the longest CAG repeat lengths had shorter AGDs. Moreover, men with an AGD above the median had a shorter CAG repeat length compared to men with an AGD below the median. However, there was no linear relationship between AGD and CAG repeat length for the overall cohort. In addition, no relationship was found between PL and AR CAG repeat length.

During sexual development, the immature genital precursors migrate ventrally via an androgen-mediated pathway. ${ }^{21}$ A marker for genital development, the AGD has been examined in both animal and humans. ${ }^{1-4}$ Investigators have also used AGD to show that agents which have the potential to disrupt androgen signaling in animal models can lead to abnormal genital lengths and even alter testicular function as measured by testosterone and sperm production. ${ }^{22-25}$

While the final determination of AGD is likely complex, androgen sensitivity likely plays a role for a subset of men. The current study demonstrated that a majority of the men with the longest CAG repeat lengths, and conceivably the most impaired androgen signaling, had significantly shorter AGDs. However, the converse was not true. Of men in the shortest quartile group of AGDs, only $12 \%$ had longer CAG repeat lengths. Thus, AR sensitivity is unlikely to be the sole factor in determining AGD, and other factors operating during the fetal period, likely through androgen-mediated pathways, also impact normal genital development. It is possible that the complex network of AGD determinants limited our ability to identify a linear relationship between AGD and AR CAG repeat length.

Several limitations warrant mention. While the AR CAG repeat length was not known during data collection, urological diagnoses such as infertility, which may correlate with CAG repeat length, were known. Thus, it was not always possible to blind observers to the men's diagnoses which theoretically can lead to observer bias. In addition, multiple observers measured genital lengths in men. While we have previously established reproducibility of measurements, variation in assessments is possible and in the current data set within and between observers, variations were not measured to minimize patient discomfort and dropout. While longer AR CAG repeat length was associated with AGD, a shorter AR CAG distance (i.e. <19 repeats) was not. Moreover, the number of men with longer CAG repeat lengths was small. Thus, the identified association with AGD may have resulted from chance alone. This becomes increasingly likely given the number of comparisons tested in Tables $\mathbf{2}$ and $\mathbf{3}$ and the absence of significant $P$ values after Bonferonni correction. In addition, a relationship with

Table 3 CAG repeat lengths stratified by AGD around the median

\begin{tabular}{|c|c|c|c|c|c|c|c|}
\hline \multirow{2}{*}{ AGD cutoff $(\mathrm{mm})$} & \multicolumn{2}{|c|}{$\leqslant$ cutoff } & \multicolumn{2}{|c|}{$>$ cutoff } & \multirow[b]{2}{*}{$\mathrm{P}^{*}$} & \multirow{2}{*}{$\begin{array}{c}\text { Multivariable analysis }^{a} \\
\text { OR }(95 \% \mathrm{Cl})\end{array}$} & \multirow[b]{2}{*}{$P$} \\
\hline & $n$ & $C A G$, mean $\pm s . d$. & $n$ & $C A G$, mean $\pm s . d$. & & & \\
\hline 36 & 81 & $22.3 \pm 3.3$ & 114 & $21.3 \pm 3.2$ & 0.06 & $1.07(0.98,1.18)$ & 0.15 \\
\hline 38 & 88 & $22.1 \pm 3.3$ & 107 & $21.3 \pm 3.2$ & 0.16 & $1.04(0.94,1.14)$ & 0.44 \\
\hline 40 & 102 & $22.3 \pm 3.3$ & 93 & $21.1 \pm 3.1$ & 0.02 & $1.11(1.01,1.22)$ & 0.03 \\
\hline 42 & 112 & $22.1 \pm 3.3$ & 83 & $21.2 \pm 3.2$ & 0.13 & $1.08(0.98,1.19)$ & 0.11 \\
\hline 44 & 124 & $21.9 \pm 3.3$ & 71 & $21.3 \pm 3.1$ & 0.39 & $1.05(0.96,1.16)$ & 0.30 \\
\hline
\end{tabular}

Abbreviations: AGD, anogenital distance; CAG, cytosine-adenine-guanine; $\mathrm{Cl}$, confidence interval; OR, odds ratio.

${ }^{a}$ Multivariable OR $(95 \% \mathrm{Cl})$ adjusted for age, race and fatherhood states the odds of having a shorter AGD for each additional CAG repeat.

* $P$ value represents Wilcoxon rank sum analyses. 


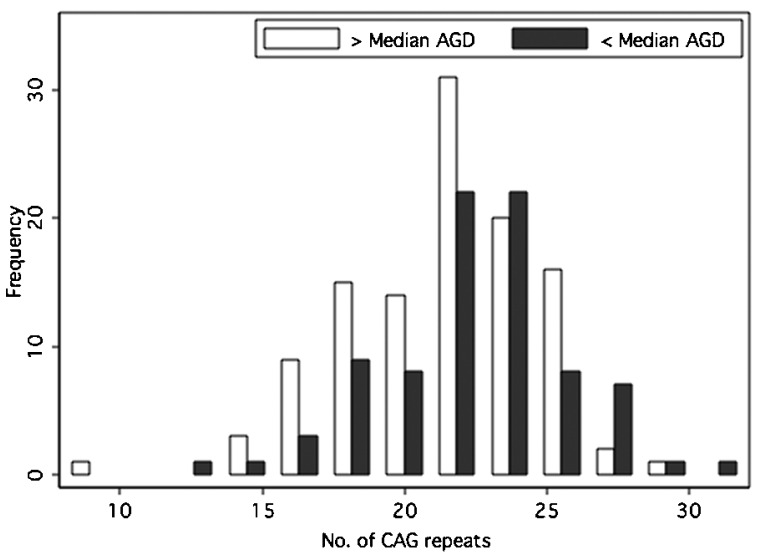

Figure 2 Distribution of CAG repeat lengths in men that had an anogenital length less than (black bars) and greater than (white bars) the median (40 mm). AGD, anogenital distance; CAG, cytosine-adenine-guanine.

AR CAG repeat number and PL was not found despite the fact that penile development is also known to be under androgen influence. It is also possible that the relative obesity in our cohort prevented accurate assessment of phallic length, which could not be overcome despite adjustment for BMI.

While no linear relationship was found between AGD and AR CAG repeat length, this is the first study to suggest a link between AGD and AR CAG repeat length. As such, AGD may provide some insight into a man's androgen sensitivity. Future studies should examine the relationship between androgen signaling and a man's CAG repeat length.

\section{AUTHOR CONTRIBUTIONS}

MLE, DJL and LIL conceived the project. MLE, TCH, AWP, MGM and RCW collected data. MLE, AWP and DJL analyzed the data. MLE drafted the manuscript. All authors provided critical revision of the manuscript.

\section{COMPETING FINANCIAL INTERESTS}

All authors declare that there are no competing financial interests.

\section{ACKNOWLEDGMENTS}

We wish to acknowledge Sandy Alexander who assisted with genital measurements.

1 Hsieh MH, Breyer BN, Eisenberg ML, Baskin LS. Associations among hypospadias, cryptorchidism, anogenital distance, and endocrine disruption. Curr Urol Rep 2008 9: $137-42$.

2 Scott HM, Hutchison GR, Mahood IK, Hallmark N, Welsh M et al. Role of androgens in fetal testis development and dysgenesis. Endocrinology 2007; 148: 2027-36.
3 Swan SH, Main KM, Liu F, Stewart SL, Kruse RL et al. Decrease in anogenital distance among male infants with prenatal phthalate exposure. Environ Health Perspect 2005 113: $1056-61$.

4 Torres-Sanchez L, Zepeda M, Cebrian ME, Belkind-Gerson J, Garcia-Hernandez RM et al. Dichlorodiphenyldichloroethylene exposure during the first trimester of pregnancy alters the anal position in male infants. Ann N Y Acad Sci 2008; 1140: 155-62.

5 Eisenberg ML, Hsieh MH, Walters RC, Krasnow R, Lipshultz LI. The relationship between anogenital distance, fatherhood, and fertility in adult men. PLoS One 2011; 6: e18973.

6 Mendiola J, Stahlhut RW, Jorgensen N, Liu F, Swan SH. Shorter anogenital distance predicts poorer semen quality in young men in Rochester, New York. Environ Health Perspect 2011; 119: 958-63.

7 Eisenberg ML, Jensen TK, Walters RC, Skakkebaek NE, Lipshultz LI. The relationship between anogenital distance and reproductive hormone levels in adult men. J Urol 2012; 187: 594-8.

8 van den Driesche S, Scott HM, MacLeod DJ, Fisken M, Walker M et al. Relative importance of prenatal and postnatal androgen action in determining growth of the penis and anogenital distance in the rat before, during and after puberty. Int $J$ Androl 2011; 34: e578-86.

9 Skakkebaek NE, Rajpert-De Meyts E, Main KM. Testicular dysgenesis syndrome: an increasingly common developmental disorder with environmental aspects. Hum Reprod 2001; 16: 972-8.

10 Skakkebaek NE, Rajpert-De Meyts E, Jorgensen N, Main KM, Leffers $\mathrm{H}$ et al Testicular cancer trends as 'whistle blowers' of testicular developmental problems in populations. Int J Androl. 2007; 30: 198-204.

11 Mifsud A, Sim CK, Boettger-Tong H, Moreira S, Lamb DJ et al. Trinucleotide (CAG) repeat polymorphisms in the androgen receptor gene: molecular markers of risk for male infertility. Fertil Steril 2001; 75: 275-81.

12 Andrew SE, Goldberg YP, Hayden MR. Rethinking genotype and phenotype correlations in polyglutamine expansion disorders. Hum Mol Genet 1997; 6: 2005 10.

13 Edwards A, Hammond HA, Jin L, Caskey CT, Chakraborty R. Genetic variation at five trimeric and tetrameric tandem repeat loci in four human population groups. Genomics 1992; 12: 241-53.

14 Chamberlain NL, Driver ED, Miesfeld RL. The length and location of CAG trinucleotide repeats in the androgen receptor $\mathrm{N}$-terminal domain affect transactivation function. Nucleic Acids Res 1994; 22: 3181-6.

15 Tut TG, Ghadessy FJ, Trifiro MA, Pinsky L, Yong EL. Long polyglutamine tracts in the androgen receptor are associated with reduced trans-activation, impaired sperm production, and male infertility. J Clin Endocrinol Metab 1997; 82: 3777-82.

16 Giwercman YL, Xu C, Arver S, Pousette A, Reneland R. No association between the androgen receptor gene CAG repeat and impaired sperm production in Swedish men. Clin Genet 1998; 54: 435-6.

17 Casella R, Maduro MR, Misfud A, Lipshultz LI, Yong EL et al. Androgen receptor gene polyglutamine length is associated with testicular histology in infertile patients. J Urol 2003; 169: 224-7.

18 Badran WA, Fahmy I, Abdel-Megid WM, Elder K, Mansour R et al. Length of androgen receptor-CAG repeats in fertile and infertile Egyptian men. J Androl 2009; 30: 416 25.

19 Hsieh MH, Eisenberg ML, Hittelman AB, Wilson JM, Tasian GE et al. Caucasian male infants and boys with hypospadias exhibit reduced anogenital distance. Hum Reprod 2012; 27: 1577-80.

20 Marcelli M, Ittmann M, Mariani S, Sutherland R, Nigam R et al. Androgen receptor mutations in prostate cancer. Cancer Res 2000; 60: 944-9.

21 Larson WJ. Human Embryology. 2nd ed. New York: Churchill Livingstone; 1997. p261.

22 Scott HM, Hutchison GR, Jobling MS, McKinnell C, Drake AJ et al. Relationship between androgen action in the "male programming window," fetal sertoli cell number, and adult testis size in the rat. Endocrinology 2008; 149: 5280-7.

23 Foster PM, Mylchreest E, Gaido KW, Sar M. Effects of phthalate esters on the developing reproductive tract of male rats. Hum Reprod Update 2001; 7: 231-5.

24 Martino-Andrade AJ, Morais RN, Botelho GG, Muller G, Grande SW et al. Coadministration of active phthalates results in disruption of foetal testicular function in rats. Int J Androl 2009; 32: 704-12.

25 Cowin PA, Gold E, Aleksova J, O'Bryan MK, Foster PM et al. Vinclozolin exposure in utero induces postpubertal prostatitis and reduces sperm production via a reversible hormone-regulated mechanism. Endocrinology 2010; 151: 783-92. 